\title{
Improved Model for Indoor Propagation Loss in the 5G FR2 Frequency Band
}

\author{
Árpád László Makara and László Csurgai-Horváth²
}

\begin{abstract}
One of the latest developments today is the 5G, or 5th generation mobile network. In addition to a number of innovations, the new system also includes millimeter-wavelength frequency ranges denoted with FR2, that formerly not applied for these specific purposes. Proper management of the transmitter and receiver antenna beams is required for efficient communication in this frequency range. For future use, the simplest implementation way is electronically shaping the antenna beams by an algorithm to orient the antennas in the best possible direction. The prerequisites for these algorithms are appropriate propagation models, which are currently lacking, and those that publicly available are not accurate enough for practical use.

Due to its complexity, solving this task is expected to be feasible only with artificial intelligence based solutions that require large amounts of input data for training. This amount of data requires long-term measurement data, which is not a feasible solution considering the rate of development. There are two possible ways to solve this: creating more accurate propagation models or apply simulations. For the latter solutions, it may also be necessary to validate based on measurement results and models.

In this paper we provide an overview of the wave propagation conditions in the new FR2 frequency range in the case of indoor use, intending to create an accurate propagation model. We present the existing models in other fields of applications and the typical propagation conditions in these bands in view of the existing research in this area. By analyzing the measurements performed at the department, we point out the shortcomings of the previous models and the parameters to be taken into account. Then we present an improved model based on the ITU recommendation for indoor propagation.
\end{abstract}

Index Terms-5G, environmental coefficient, FR2 band, indoor propagation, path loss model

\section{INTRODUCTION}

$\mathrm{T}$ HE next generation of wireless networks is the 5th generation mobile network, for which some of the standards are still being developed today. The need to develop a new system is caused by the drastic increase in consumer demand [1], based on current trends. The number of devices on the network simultaneously is continuously growing, requiring a faster and higher capacity network creation.

With regard to the standardization processes, it is worth saying a few words about the bigger and more significant organizations. The 3GPP (3rd Generation Partnership Project) umbrella organization brings together the various

${ }^{1,2}$ Department of Broadband Infocommunications and Electromagnetic Theory, Budapest University of Technology and Economics, Hungary.

1'(e-mail: makara.arpadlaszlo@edu.bme.hu)

2(e-mail: csurgai-horvath.laszlo@vik.bme.hu) standardization processes of mobile communication. The ITU is the UN specialized agency whose task is international facilitating telecommunications cooperation. In this work, IMT-2020 (International Mobile Telecommunications-2020) standard was the guideline for specifications and standards [2].

\section{A. $5 G$ system requirements}

In $5 \mathrm{G}$ a number of new uses are planned, based on past trends [1]. With the new system for wireless connections in existing areas a drastic increase in speed would be achievable. Without wishing to be exhaustive, the main areas of application are: [2]:

- Support for real time video calls.

- High-speed data connection support (50 MB/s or higher).

- Serving high-speed users (up to $500 \mathrm{~km} / \mathrm{hr}$ ).

- IoT support (such as wireless operation of sensor networks).

- Ultra-reliable communications (URC).

- Massive machine type communications (mMTMC).

- Enhanced mobile broadband (eMBB).

In fact, to meet user needs (existing and future) requirements for the $5 \mathrm{G}$ network [3], assumed increasing number of assets necessitated the designation of new frequency bands as listed in Table I:

TABLE I

5G REQUIREMENTS

\begin{tabular}{ll}
\hline \hline User data speed & Downlink: $100 \mathrm{Mbps}-1 \mathrm{Gbps}$ \\
Maximal data speed & Uplink: $50 \mathrm{Mbps}-500 \mathrm{Mbps}$ \\
Bandwidth & Downlink:20 Gbps; Uplink: $10 \mathrm{Gbps}$ \\
Mobility & Up $\mathrm{MHz}-1 \mathrm{GHz}$ \\
& Ultra-Reliable and Low Latency \\
Delay & Communications: $0.5 \mathrm{~ms}$ \\
& Enhanced Mobile BroadBand: $4 \mathrm{~ms}$ \\
Connections density & $250.000 \mathrm{user} / \mathrm{km}^{2}$ \\
Local traffic capacity & $15 \mathrm{Mbps} / \mathrm{m} 2$ \\
\hline \hline
\end{tabular}

The new frequency bands used previously only for satelliteto-Earth communications or in the access network of the mobile communications system. The characteristics of these new domains are different for mobile communication bands so far used, so their empirical description is particularly important for implementation.

\section{B. The frequency range examined}

One of the many designated frequency bands is $38 \mathrm{GHz}$ (and vicinity), which is the main subject of this paper. However, the statements made are similar to the other higher frequency ranges. The signal propagation in the investigated band has a different nature than the traditional lower frequencies. In 
addition, it is no longer negligible that solid bodies are also have significant attenuation effect [4]. Among other things, this is why we can talk about different indoors and outdoors models.

In such a high frequency range, the transmitter and receiver antenna beam orientations are particularly critical [5] for good reception conditions, in fact, for as long as a device is connected to the network. In many application areas the receiver moves relative to the transmitter while connected to the network. But even if both the transmitter and the receiver are in a constant, fixed position, a change can and will happen in the space between them. For all these reasons, it is essential that at least in the receiver, be able to control the antenna beam, thus provide better reception conditions [6]. However, the time required for control should be as short as possible, regardless of outdoor or indoor use, and no user can feel that there is significant fluctuation in the received signal level.

Summarizing all this, it becomes necessary to change the operational environment as quickly as possible by adaptive beamforming, in order to maximize the transmitter and receiver antenna directing at each other, over the entire range of operating time. The beamforming control must be such that the user does not take anything from it and the quality of service also meets the expectations.

The complexity of the task is predicted to require an adaptive beamformer solution based on artificial intelligence algorithms in the future application of higher frequency ranges. With all this, the goal is to ultimately produce a model with which the necessary beamforming algorithms will be trainable for future use.

\section{The structure of the paper}

In Section 2, we present the frequency ranges of the $5 \mathrm{G}$ radio interface. Following this, in Section 3, we describe the various public propagation models, highlighting them separately the one that is best used for our purpose.

After all, we will briefly summarize how the department developed the competence required for the task in Section 4 and what tools are available to us to perform the measurements. Next, in Section 5, we analyze the completed measurements and compare these results with the relevant models and show what further steps are taken necessary for the end use. Finally, in Section 6, we present a new model designed to facilitate adaptive beamforming based on the experience shared in the previous chapters.

\section{5G FREQUENCY BANDS AND THEIR CHARACTERISTICS}

5G NR (Abbreviation for 5G New Radio) [8] stands for layer radio access technology (standard abbreviation: RAT) [9]. The $5 \mathrm{G}$ NR defines two frequency bands, and several within subbands:

- $\quad$ Frequency Range 1 (FR1): Bands below $6 \mathrm{GHz}$

- $\quad$ Frequency Range 2 (FR2): Between 24.25 and $52.5 \mathrm{GHz}$

There are also currently plans to expand the FR1 range to $410 \mathrm{MHz}$ and $7125 \mathrm{MHz}$ and that, in the case of FR2, bands up to $100 \mathrm{GHz}$ should be established. How much bandwidth will eventually be needed will be determined by the user needs.

In 2018, the n260 has already been allocated for use in the US (among other things) [10]. In the case of Hungary, during 2019 they were only allocated in FR1 bands for the operation of $5 \mathrm{G}$ [11]. The mobile service providers that have won these bands are already advertised as an available service.

This paper covers the frequency range of $38 \mathrm{GHz}$ and surrounding in the FR2 band for a more thorough examination.

\section{A. Path loss in FR2 frequency bands}

When using $5 \mathrm{G}$, the radio waves will in most cases propagate in the air, respectively sometimes through walls, objects, but ultimately in a dominant path in open space. The relative dielectric constant of air is the function of frequency, temperature, pressure and humidity [12] (only the very first parameter has an actual influence). For outdoor use, this means that this frequency band is already highly exposed to the weather [14]. From a practical application point of view, the attenuation of the medium will change, which will be proportional to the distance [12].

Another significant factor besides attenuation is the occurrence of reflections (related to even the issue of transmission, but this is now secondary). The electromagnetic wave reflected at a certain percentage at the medium boundary. The degree of reflections is basically affected by material properties or the frequency, while the physical size of the reflecting object must be larger than the wavelength.

It is important to talk about interference on the topic of reflections. Interference occurs when two or more waves meet and their phases are different. We can speak of constructive or destructive interference, depending on whether the amplitude of the wave (signal) will be larger or smaller than it was originally. Where there is reflection, there is a high chance of interference that is greater it is likely to be destructive rather than constructive.

\section{B. Uses of the $38 \mathrm{GHz}$ band to date}

The department has former experience with this frequency band for Alphasat (Inmarsat-4A F4) [15] to communicate with a satellite and during these the main propagation characteristics were examined. A feature of this application is that the exact position of the satellite is always known, so the antennas are directed at each other without any particularly great obstacle in the path. The most important factor of the mode of use is the individual antennas orientation [5]. In terms of practical implementation, it is typically narrow in direction antennas with high gain are used.

In addition to satellite reception, the department has previously conducted a shorter research on $38 \mathrm{GHz}$ band. In 2018 [7] we examined how behave electromagnetic waves at this frequency indoors, including in cases when antenna orientation to each other was not ensured. Measurements related to the project results were compared with ITU-R Recommendation P.1238 [16], during which it is concluded that the recommended model gives an acceptable result as long as antenna orientation towards each other is ensured. 


\section{Existing Propagation Models}

The models can be divided into three large groups: indoor, outdoor and free space. In addition we can distinguish between empirical, deterministic, and semi-empirical models.

For empirical models a correlation based on a statistical approach can be given. Easily and are quick to use, in return they are not always the most accurate. In a deterministic case based on some preliminary model, the propagation in the given space is calculated to get the quantity sought.

For mobile communication networks, outdoor and indoor models are important, however, outdoor propagation models can also be used for a kind of control (but these are best for point-to-point connections in particular).

The number of models dedicated to this frequency band is very low in the literature. Due to the nature of the problem, deterministic modelling methods are accurate but require more computing capacity, while the ray-tracing methods can be used along affordable computational capacities [13].

From end use, it follows that we want to perform as few calculations as possible in order to get results as soon as possible. In this paper, we deal only with the examination of empirical models.

\section{A. Free-space propagation}

In the case of outdoor propagation, most models assume direct vision or other special circumstances. The attenuation per unit length thus calculated is typically lower as for indoor propagation models, but of a similar order of magnitude [17]. Hence an estimate can be used in indoor modelling (taking into account its limitations). The most common such relationship, which we simply refer to as outdoor attenuation hereinafter [21] takes the following form:

$$
P_{L}=20 \cdot \log _{10}(d)+20 \cdot \log _{10}(f)+92.45
$$

where $d$ is the distance in kilometres, $f$ is the frequency in $\mathrm{GHz}$, and the result is given in decibels and describes the outdoor attenuation between isotropic antennas in vacuum. Once the medium in which the wave propagates is already air, additional attenuation occurs up. For low distances Fig. 1. depicts the path loss calculated with Equation (1).

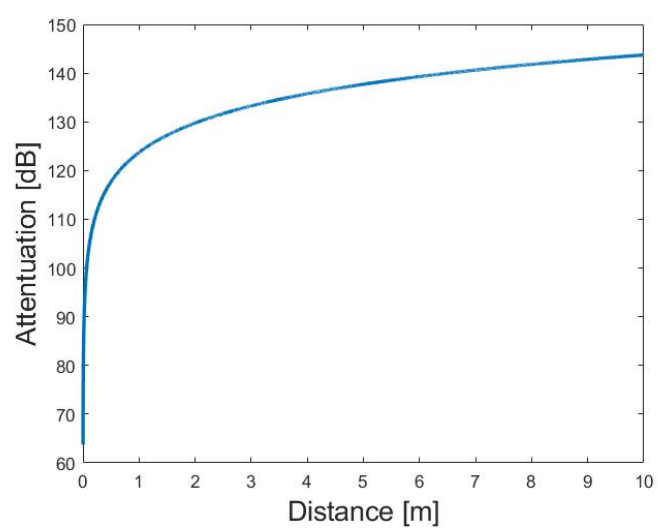

Fig. 1. Short-range free-space path loss in FR2 band at $38.72 \mathrm{GHz}$

\section{B. Close-in free space reference distance path loss model}

The Close-in free space reference distance path loss model [22] (hereinafter CI) is a reference model based on outdoor propagation and it is applied for comparison multiple indoor signal propagation results:

$$
P_{L}^{(C I)}=\operatorname{FSPL}\left(f, d_{0}\right)+10 \cdot n \cdot \log _{10}\left(\frac{d}{d_{0}}\right)+X_{\sigma}^{C I}
$$

where $\operatorname{FSPL}\left(f, d_{0}\right)$ is the outdoor attenuation at the reference distance ( $d_{0}$ is 1 meter) at the given frequency $f$ measured in $G H z, n$ is the path loss factor (PLE) and $X_{\sigma}^{C I}$ is a zero-valued Gaussian random variable with $\sigma$ standard deviation. The measured PLE [23] coefficients found in the literature are largely the same in the ITU model of indoor signal propagation with distance-based loss coefficients. A semi-outdoor, semiindoor measurement can be found in the literature where the measured PLE is double of the ITU model, however, a high value for standard deviation was measured here [24].

\section{Outdoor propagation models}

Outdoor propagation models usually differentiate cases according to how they are built and whether the area is environmental or natural. In addition, one can count on individual models on topography, degree of incorporation, location of the transmitting antenna, climate characteristics and other similar factors. In a sense, the simplest such model is the ITU surface model [25]. Of interest for classification are models that are essentially outdoor, but are used in a somewhat enclosed built environment. In the literature can be found a measurement procedure (and, in fact, a result) that is a corridor open from one side were thus considered to be predominantly outdoor measurements [24].

One of the most common relationships describing outdoor propagation is the Okumura model [23], in the following form:

$$
P_{L}=l f+A_{m u}(f, d)-G_{(h t e)}-G_{(h r e)}-G_{\text {area }}
$$

where $P_{l}$ is the attenuation, $l f$ is the free-space attenuation, $A_{m u}(f, d)$ is the relative median attenuation outdoors (as a function of frequency and distance), $G_{(h t e)}$ is the transmitter gain, $G_{(h r e)}$ is the receiver gain, $G_{\text {area }}$ is the amplification of the environment. The model is actually breaks down the attenuation into two parts: into an outdoor component and factors that modify the environment, and to the amplifying of the environment (which can even be attenuation).

An improved version of the Okumura model is the Hata model [22], which distinguishes the outdoor locations depending on built-in rate.

The COST-Hata model [23], (a further development of the Hata model) takes the following form:

$$
\begin{aligned}
P_{L}=46.3+33.9 & \cdot \log _{10}(f)-13.82 \cdot \log _{10}\left(h_{B}\right) \\
& -a\left(h_{R}, f\right) \\
& +\left(4.9-6.55 \cdot \log _{10}\left(h_{B}\right)\right) \\
& \cdot \log _{10}(d)+C_{m}
\end{aligned}
$$

where $P_{L}$ is the median loss/attenuation, $f$ is the frequency in $M H z, h_{B}$ is the transmitting antenna effective height in meters, $d$ is the distance in $k m, h_{R}$ is the effective height of the (mobile) receiving antenna in meters, $C_{m}$ is the constant offset in $d B$. 
The model distinguishes through the parameter $a\left(h_{R}, f\right)$ whether the propagation is urban or suburban.

\section{Indoor propagation models}

Indoor propagation models are the most important for this paper, as our primary goal is to create an improved model describing indoor propagation. The main feature is that the receiver area surrounded by walls, that represent significant attenuation for the passing wave. In addition, they are located at relatively short distances for some antennas, the air composition does not change for a significant part of the time (or up to negligible extent), but moving people and living things can occur [18]-[20]. In general it can be said that most models were originally for lower frequency bands made. In recent years, they extended for higher frequency bands (such as ITU indoor propagation model), upon different considerations [16], [24], but few models are available for such high frequency ranges (mainly empirical).

Starting from the COST-Hata model, the TYM model [23] was created, which is already indoor applicable, the results are consistent with the measurements can be obtained for the $38 \mathrm{GHz}$ band. The COST-Hata model served as a basic idea for constructing the TYM model. The studied model takes into account the effects of temperature and humidity based on the signal propagation properties:

$$
\begin{gathered}
P_{L}=96.7-0.562 \cdot S_{\text {win }}-0.3666 \cdot f+0.092 \cdot d \\
-0.187 \cdot h+0.257 \cdot t
\end{gathered}
$$

where $P_{L}$ is the loss, $S_{\text {win }}$ is the window size (at a given level the average window size, which is expressed by the surface of the window), $f$ is the frequency, $d$ is the distance (in meters), $h$ is humidity and $t$ is the temperature.

The biggest flaw of the model, in terms of practical use, is that of windows the average surface area on a mobile endpoint is not measurable or only impermissibly large at the cost of effort.

The ITU's empirical formula for indoor signal propagation is as follows [24]:

$$
L_{\text {total }}=L\left(d_{0}\right)+N(f) \cdot \log _{10}\left(d / d_{0}\right)+L_{f}(n)
$$

where $L\left(d_{0}\right)$ is the basic transmission loss at a reference distance $\mathrm{d}_{0}=1 \mathrm{~m}, N$ is the distance based loss factor, distance $d$ in meters with a minimum value of 1 meter, $L_{f}$ "floor" transit loss factor in $d B$ and $n$ is the number of floors crossed. Based on the measurement results presented in Section 5 and the literature, in simple, managed cases, the ITU model gives an acceptable result. If this is not the case above, we can experience a deviation of the order of $50 \mathrm{~dB}$. There is a high degree of similarity between the ITU and CI models (Equations (6) and (2), respectively) as it can be seen at the formula level. The ITU model can actually be considered an averaged CI model, which was expanded with slabs intersected by the signal path.

Because of all this, the conclusion is that the initial model is hereinafter the ITU indoor propagation model, as it is the simplest and has only such parameters, which can be easily measured by a well-equipped receiver.

\section{The Measurement SEtuP}

Under the frame of ESA's (European Space Agency) technology transfer program the Alphasat communication experiment's ground receiver system's elements were applied in a terrestrial, indoor propagation measurement system, designed for the Q-band. BME-HVT in collaboration with Totaltel Telecom Techniques Ltd. [7] developed the Q-band downconverter unit for the experiment.

The receiver for the indoor measurement setup is originated from the Alphasat ground receiver station of the department. The transmitter and receiver are made up of two directional horn antennas assembled on a motorized tripod with rotating platform. The $360^{\circ}$ horizontal rotating capability allows scanning the entire horizontal plane and recording of the received signal power parameters for a specific location as shown in Fig. 2. The local clock frequency was generated by an external PLL synthesizer board [7], operating at $9.35 \mathrm{GHz}$. A frequency quadrupling in the receiver/transmitter site results the Q-band carrier signal. The intermediate frequency (IF) signal is an unmodulated sine wave at $1.32 \mathrm{GHz}$, therefore the exact transmit frequency is $4 \cdot \mathrm{CLK}+\mathrm{IF}=38.72 \mathrm{GHz}$. The fixed transmitter and the rotated receiver configuration allow measuring both Non-Line of Sight (NLOS) and Line of Sight (LOS) scenarios.

With a systematic relocation of the receiver, a signal strength map of the room can be provided by the angular dependency of the signal to be measured. In order to measure and process the received signal a Software Defined Radio (SDR) platform is utilized. The SDR-based data collection and the platformcontrolling software was developed by other colleagues at the department as it was published in [7]. The data collection took place in a GNU radio environment. The measurement data were generated by rotating the receiver every five degrees, which were processed with MATLAB. Measurements were made in a total of five different rooms, but due to lack of space, only one of them is presented in this article.

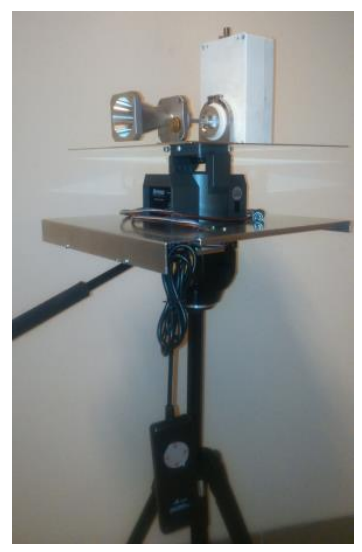

Fig. 2. Receiver on motorized platform with horn antenna and downconverter

The SDR acquires the measurement of incoming and downconverted L-band signal. After digitization the whole signal processing will be performed by the controlling computer using the GNU Radio software platform [7]. 
There is a computer responsible for controlling and managing the SDR. This is an essential issue, because during the measurement human interaction is minimized as much as possible, since the millimeter wave frequency band is sensitive to humans in the measurement area, and this significantly influences the result. The signal reflections and interferences in this band can considerably modify the propagation environment.

\section{MEASUREMENT Results}

Each measurement was performed in the BME V1 building. The affected parts of the building, respectively in the rooms we were locked out of the citizens of the university at the time of the measurement, so that the environment does not change in any way. Persons performing the measurements are always as far away from the antennas as possible.

One of the measurement sites was room 103 of the V1 building, which is on the first floor of the five-storey building and our measurements and methods are presented here with through this location. Fig. 3. shows the floor plan of the room. The measurement points are designated by each $\mathrm{x} / \mathrm{y}$ format point, a TX is the transmitting antenna. At the top of the figure we indicate the windows and some furniture are also shown. The second measurement site was a classroom on the fifth floor of the building, and it has two sides with entirely plastic framed windows.

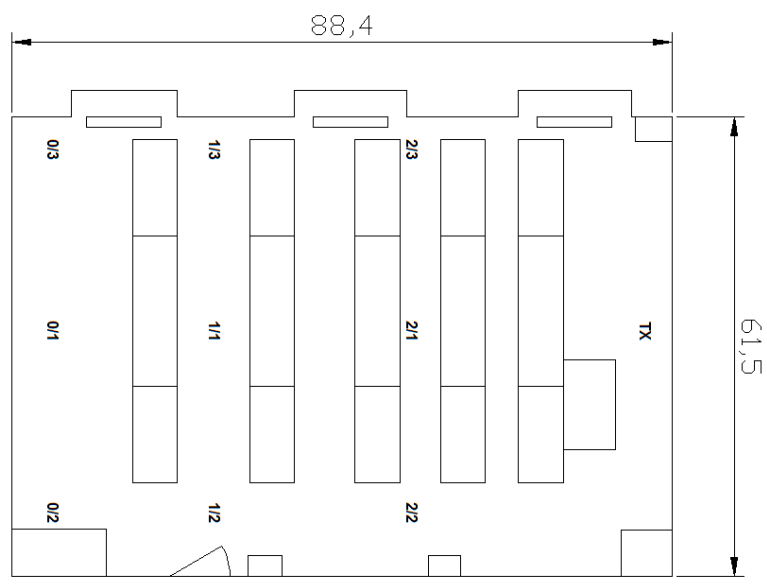

Fig. 3. Floor plan of room 103 in building V1 and the measurement points

Both the transmitter and receiver were located 1.5 meters high. On the moving platform, the receiver turned every 5 degrees, a positive detour direction, thus 73 measurement results were recorded at each position. The transmitter was always pointing toward the measured receiver location. As an example, Fig. 4. depicts a typical measurement where several reflections are observable besides the main lobe.

After analysing the results, the conclusions are the following:

- The received signal level is the function of distance, the angle of the antenna beams and the reflective surfaces around.

- At this frequency, the glass surfaces have a large angle of incidence they reflect.
- Even if the transmitter looks at the receiver, depending on the latter's direction there are usually two or three possible acceptable signal levels from reflected pathways.

- With a few degrees of rotation, the received signal level may decrease by $40-60 \mathrm{~dB}$.

- The signal level (according to previous models) decrease by several $\mathrm{dB}$ on every few meters.

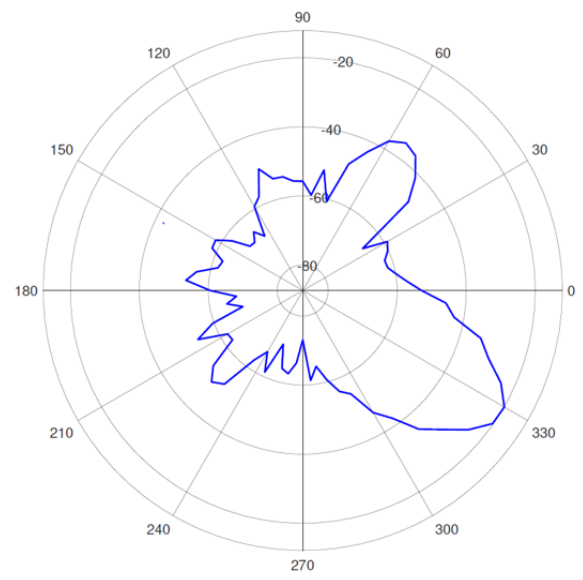

Fig. 4. A measurement result with high signal level reflected components

In Fig. 5. the measurement location is shown, where the transmitter is on the left side of the picture, while the receiver on the right side.

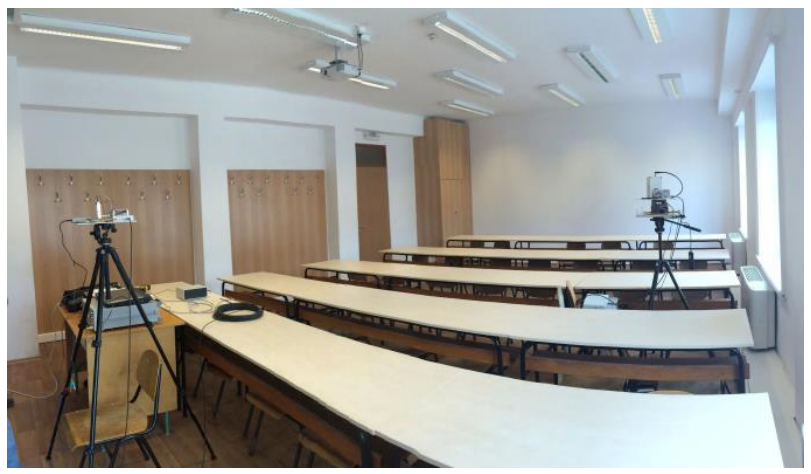

Fig. 5. Transmitter and receiver setups in the measurement location

Based on the results so far, it is appropriate to introduce a relative environment concept. By this is meant that the signal level that can be received at a given point depends on the distance and the reflection environment. For the latter, no matter how far there is a point from the transmitter, all that matters is the nature of the environment.

According to this the individual receiving points can be typified, which can be called relative environments, since ultimately, they specify the nature of the beamforming strategy required. The distance will only determine the maximum signal strength. With the concept thus created each acquisition point can be categorized as needed during the modelling. 


\section{AN IMPROVED INDOOR PROPAGATION MODEL}

To develop a new model, the main goal was to find the simplest solution that acceptably fits the measurement results and takes into account the physical factors influencing the spatial distribution. One of the possible alternatives the empirical expansion of the ITU indoor propagation model seemed to be the best choice. The following conditions can be formulated in advance:

- The measured values are considered correct, assuming only a small error.

- The main parameters should be the distance and the angle between the transmitter and receiver antenna directions.

- Work with as few parameters as possible.

- The parameters should be included in the simplest possible functional relationships.

On the basis of current measurement results and according to the literature [25], in NLoS situations (but more generally in all non-LoS cases) the attenuation behaves as if we were adding an additive term to the outdoor attenuation value. Of course, in reality, the process more complicated than this, however, in terms of empirical modelling, this approach is acceptable.

As a starting point, we used the ITU model for indoor propagation. The reason is that this model describes quite precisely the attenuation between the antennas directed at each other. The following model was found to be the most suitable for the previously stated goals in terms of use:

$$
L_{\text {total }}=63.76+20.3 \cdot \log _{10}(d)+F(x)+G(\alpha, \beta)+C
$$

where $d$ is the distance in meters, $F$ is the relative environment as a function of attenuation and $x$ is the relative environment in which it resides, $G$ is the attenuation due to the directivity of the two antennas and constant $C$ is the specification of the ITU recommendation constant.

The model formulated in this way leads to a regression calculation problem, where an algorithm using an iterative learning method can determine the coefficients.

The problem was solved by regression calculations. In doing so, LMS (Least-Mean-Square) error criterion was applied, using the instantaneous gradient method. Denote by $F$ an error function whose global minimum we are looking for [26]-[27]:

$$
\hat{F}=[t(k)-a(k)]^{2}=e^{2}(k)
$$

where $k$ is the current iteration number, $t$ is the target, i.e. the expected output, and $a$ is the approximate value of the function in the given iteration. The error $e$ is the difference between the expected and the calculated value. That is actually can be considered as supervised machine learning, where for specific input parameters the expected output is known. By the end of the process, the algorithm learns in the examined frequency band the coefficients of the empirical model. Using the stochastic gradient method (which converges the fastest to a minimum), the following result is obtained [27]:

$$
\nabla \hat{F}(k)=\nabla e^{2}(k)
$$

It can be deduced that in the case of LMS we get the following form [26]:

$$
\vec{W}(k+1)=\vec{W}(k)+2 \cdot \mu \cdot \vec{e}(k) \cdot \vec{p}(k)
$$

where $\vec{W}$ is the vector of the weights to be updated, constants of $F, G, C$ members in Equation (7), and $\vec{e}$ is the error vector, which in this case will be one-dimensional. $\vec{p}$ is the input parameter vector, which in the model is a distance, $\alpha$ is the localization, and $\beta$ and constant $l$ for the $C$ term. The learning rate is less than 0.5 for this method in order to keep the method stable [26].

Here, in addition to the constants, we also have to talk about the function relationship with the input parameters. From the point of view of the implemented algorithm, it is important to mention the possibility of batch training. As the probability of occurrence of the parameters is equal, the run can be accelerated by combining the results of $N$ iteration steps.

\section{A. Parameter estimation}

Another important factor in relation to each parameter is the type of functional relationship with the input parameters. Considering the angular relationship, we made an assumption that the transmitter is directed by an acceptable extent to the receiver, so the difference in angles is due to the fact that the receiver is not well oriented. Classic transmitting antennas (when the transmit antenna is wide-beam), this model is completely correct. In case the transmitter has some kind of MIMO layout (for which the $5 \mathrm{G}$ specification gives the possibility [2]) the problem goes beyond this simple model, so it cannot be used for it.

Considering the "classic" case, the main emphasis was on keeping the angle as small as possible close between the two antennas, the more accurate is the model, and yet the simplest functional relationship is existing for faster computation.

The conclusion is that we are looking for the connection in the form of $D \cdot \sqrt{\beta}$ where $D$ is the constant sought, closed by the angle $\beta$ with the receiver, as they are typically root functions on those that are simple and have such properties.

Parameter $C$ is to estimate the antenna beamwidth, also included in the ITU recommendation parameter and it can be refined, based on the measurement results.

\section{$B$. The relative environmental coefficient}

The relative environmental coefficient is the amount we introduced for how it could be to squeeze as much information about the environment into a constant and additionally it is possible to insert into the model and can be determined by measurement. We characterize the previously defined relative environment with a single coefficient at given moment and point. This constant will always be a function of the current direction of the transmitter.

The constant (denoted by $R K$ ) can be approximated as the quotient of two integrals:

$$
R K=\frac{\int_{0}^{2 \pi} f(\phi, k) d \phi}{\int_{0}^{2 \pi} g(\phi) d \phi}
$$

where $R K$ is the relative environmental coefficient, $f(\phi, k)$ is 
the set of points above the level $k$, while $g(\phi)$ are values for the entire range, as represented visually in Fig. 6 . The result of the quotient is a number between 0 and 1 .
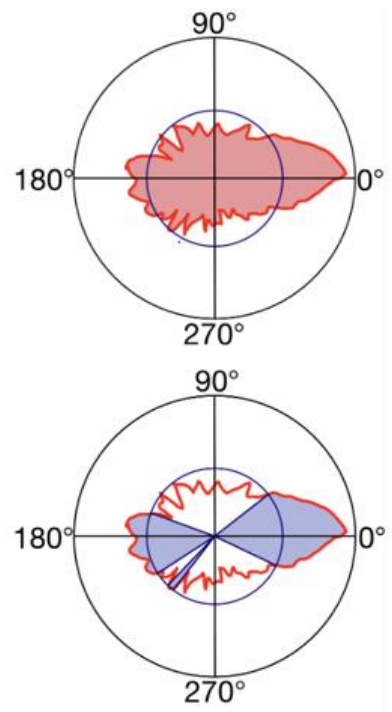

Fig. 6. Visual representation of Equation (11) for a general case. Top figure is the numerator, bottom figure is the denominator interpretation.

From a practical point of view, the choice of the coefficient $k$ is critical. It can also be tied to a specific fixed signal level that expresses what is the minimum that it is necessary to achieve in order to measure, but it can also mean what the level is already important or confusing to us. To do this, we made the following consideration:

- If a dominant signal level exists, compute only those that are comparable to and even if there are several orders of magnitude smaller peaks, we neglect them.

- If there is no dominant signal level or there are several similar ones, avoid all of them into the calculation and all that is comparable to this.

- Unevenness of noise and error during measurement should not be included in the constant.

To solve this, a constant tied to the maximum signal level was obtained to best fit the problem. In terms of measurement, it is necessary to estimate the relative environmental coefficient from finite number of measurements. It is important that each measurement should always be made in a different direction, no two measurement locations should match, taking care to map the entire space as much as possible. Then let's get closer to integration with small $d$ elements, which expresses that not at every point, but some (not necessarily equal) by closing small angles in each direction a measurements along a complete circle:

$$
\begin{gathered}
\widehat{R K}=\frac{\sum_{n=1}^{N} f(n, k) \cdot d \phi}{\sum_{n=1}^{N} g(n) \cdot d \phi}=\frac{\sum_{n=1}^{N} f(n, k)}{\sum_{n=1}^{N} g(n)} \approx \widehat{R K}_{b} \\
=\frac{\sum_{n=1}^{N} t(n, k)}{\sum_{n=1}^{N} n}
\end{gathered}
$$

where $\widehat{R K}$ is the estimated coefficient and its approximated value $\widehat{R K}_{b}$ can be quickly calculated numerically, $t(n, k)$ is a function above the corresponding signal level, which takes the following form (where $t$ is a characteristic or indicator function, $n$ is the measuring point):

$$
t(n, k)=\left\{\begin{array}{l}
1, \text { if } f(n)>k \\
0, \text { otherwise }
\end{array}\right.
$$

In determining the approximate value $R K_{n}$ we used the assumption that a significant part of the calculated area will be above the specified $k$ level, so we can simplify all of this in both the denominator and the numerator. According to experience, this is correct in practice and both solutions gives sufficiently accurate results.

Applying the numbers thus obtained to the room 103 of V1, we obtain Fig. 7. with which the model is perfectly functional and we get a result close to the measured value.

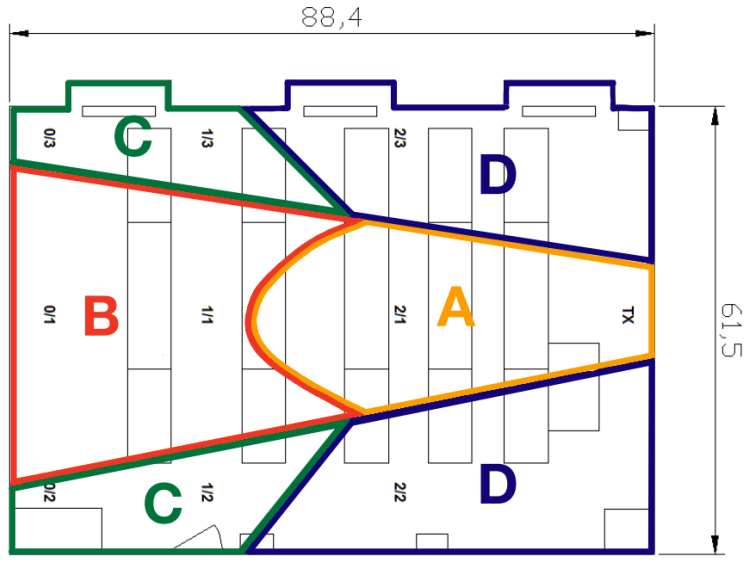

Fig. 7. Relative environmental coefficients in room 103, building V1

The model obtained by the procedure works well along the boundary conditions presented earlier. It can be used to calculate the maximum signal level that can be received at the individual points for known environment and the receiving antenna signal drop can be estimated resulted by the unalignment. On the other hand, with the estimated parameters required for the model is able to calculate the maximum value that belongs to the angle at which the beam of the transmitter and the receiver are directed to each other and are able to correct themselves based on this. The model is also functional in the inverse role of angles. As a result of the calculations, the new model takes the following form:

$$
L_{\text {total }}=63.76+20.3 \cdot \log _{10}(d)+15 \cdot R K+25 \cdot \sqrt{\beta}+C
$$

where $\beta=0$ radians is the direction in which the receiver is facing the transmitter directly with its beam and, by definition, may be $\pi$ at most magnitude, its sign always being positive. $\mathrm{C}$ is the antenna beamwidth correction term.

Fig. 8. shows the difference expressed in $[\mathrm{dB}]$ between the measurement results and the individual models in room 103. For the ITU model, the deviation is between 0 and $40 \mathrm{~dB}$ (upper figure). For our model, the values are between -4.5 and $6 \mathrm{~dB}$. Similar results were obtained for other measurements in different rooms. 


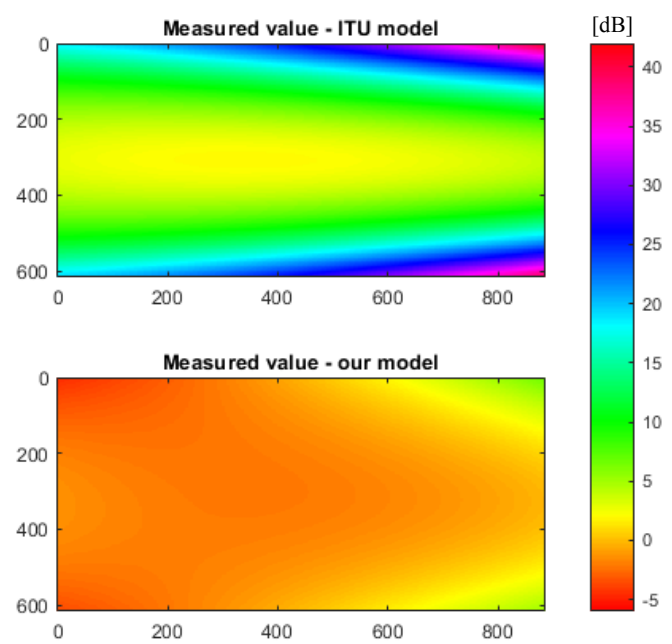

Fig. 8. The difference between the measured results and the values for both models in room 103, building V1

Thus, the average absolute error is calculated to be greatly reduced. The average absolute error during a given measurement is nearly $70 \%$ smaller (by more than $80 \%$ in some cases) comparing it by the ITU model.

The model created in this way is suitable to describe the propagation conditions of a real room. This way a large amount of data can be easily generated to train a machine learning algorithm to control the antenna beam direction, that is a desirable goal in 5G technology. However, the algorithm should be still improved since the initial model only describes simple, static problems. A future direction of this research is to implement these algorithms and prove their applicability in real environment.

\section{CONCLUSION}

Possibilities and limitations of using higher frequencies in connection with $5 \mathrm{G}$ developments were known already before. Several reports can be found in the literature and measurement concluded that the antennas were directed at each other critical to a proper radio connection. However, the models available so far (which are relatively few) only describe cases where this condition is met. This is a main problem in the practical introduction of these higher (FR2) frequencies, since an unresolved issue on the FR2 bands is adaptive beamforming. In the course of this work, by processing the measurement results, we successfully prepared an angle-dependent model. This proves that a model for the frequencies in question can be created and based on this the beamforming control is feasible. The new model only includes parameters that by an average receiver can be measured. In addition, a more useful application option is that by using the model the results of Ray-Tracing procedures can be validated without measurements obtained. Artificial intelligence may already be sufficient for the resulting data set, which makes the problem much more solvable in real situations and makes the use of FR2 frequencies available to a wide range of users. As a further plan, it arises to refine the model and use the necessary one to create algorithms.

\section{ACKNOWLEDGMENTS}

The research reported in this paper and carried out at the Budapest University of Technology and Economics has been supported by the National Research Development and Innovation Fund based on the charter of bolster issued by the National Research Development and Innovation Office under the auspices of the Ministry for Innovation and Technology.

The authors also appreciate the support on SDR-based data collection system for Dr. Bálint Péter Horváth in the Digital and Optical Communications Systems Laboratory and the cooperation with Ericsson Hungary in this research field.

\section{REFERENCES}

[1] R. E. Hattachi and J. Erfanian, "NGMN 5G White Paper," Final Deliverable, 5G Initiative Team, Feb. 2015.

[2] ITU, "International Mobile Telecommunications-2020 (IMT-2020 Standard)," tech. rep., 2019.

[3] T. Norp, "5G service requirements." https://www.3gpp.org/newsevents/3gpp-news/1831-sa1, Accessed on 21.11.2020.

[4] D. M. Pozar, "Microwave Engineering; Introduction to Microwave Systems.” John Wiley \& Sons, Inc., N.J., third ed., 2005.

[5] ITU-R Recommendation V.431-8, "Nomenclature of the frequency and wavelengh bands used in telecommunications." 08/2015.

[6] T. S. Rappaport, S. Sun, R. Mayzus, H. Zhao, Y. Azar, K.Wang, G. N.Wong, J. K. S. Schulz, M. Samimi, and F. Guitierrez, "Millimeter Wave Mobile Communications for 5G cellular: It Will Work!," IEEE Access, vol. 1, 2013.

[7] L. Csurgai-Horváth, B. Horváth, I. Rieger, J. Kertész, and B. AdjeiFrimpong, "Indoor Propagation Measurements for 5G Networks," 11th International Symposium on Communication Systems, Networks \& Digital Signal Processing (CSNDSP), 2018. DoI: 10.1109/CSNDSP.2018.8471761

[8] Erik Dahlman, Stefan Parkvall, Johan Skold, "5G NR: The Next Generation Wireless Access Technology,” Elsevier Ltd. 2018.

[9] M. Säily et al., "5G Radio Access Network Architecture for Terrestrial Broadcast Services," in IEEE Transactions on Broadcasting, vol. 66, no. 2, pp. 404-415, June 2020, DoI: 10.1109/TBC.2020.2985906.

[10] Y.Wang, J. Li, L. Huang, Y. Jing, A. Georgakopoulos and P. Demestichas, "5G Mobile: Spectrum Broadening to Higher-Frequency Bands to Support High Data Rates," in IEEE Vehicular Technology Magazine, vol. 9, no. 3, pp. 39-46, Sept. 2014, Dor: 10.1109/MVT.2014.2333694.

[11] National Media and Infocommunications Authority (NMHH), "Documentation of the Auction Procedure Announced for Frequency Use Entitlements Related to Wireless Broadband Services Specially Supporting the Introduction of 5G,", June, 2019.

[12] F. W. Vook, E. Visotsky, T. A. Thomas and A. Ghosh, "Performance characteristics of 5G mmWave wireless-to-the-home," 2016 50th Asilomar Conference on Signals, Systems and Computers, Pacific Grove, CA, 2016, pp. 1181-1185, Dor: 10.1109/ACSSC.2016.7869558.

[13] F. Hossain, T. K. Geok, Tharek Abd Rahman, and M. N. Hindia, "Indoor Millimeter-Wave Propagation Prediction by measurement and Ray Tracing Simulation at 38 GHz,’ Symmetry, vol. 10, 2018.

[14] H. Xu, T. S. Rappaport, R. J. Boyle, and J. H. Schaffner, "38-GHz Wide-Band Point to-Multipoint measurements Under Different Weather Conditions," IEEE COMMUNICATIONS LETTERS, vol. 4, p. 2, 2000.

[15] G. Codispoti et al., "The propagation and telecom experiments of the Alphasat Aldo payload (TDP5 Q/V band experiment)," 6th European Conference on Antennas and Propagation (EUCAP), Prague, 2012, pp. 116-116, DoI: 10.1109/EuCAP.2012.6206723.

[16] ITU-R Recommendation P.1238-9, "Probability distributions relevant to radiowave propagation modelling," ITU, 2001.

[17] H. T. Friis, "A Note on a Simple Transmission Formula," in Proceedings of the IRE, vol. 34, no. 5, pp. 254-256, May 1946, DOI: $10.1109 / J R P R O C .1946 .234568$. 
[18] S. Sun, T. S. Rappaport, A. T. Timothy, G. Amitava, C. N. Huan, Z. I. Kovács, R. Ignacio, K. Ozge, and P. Andrzej, "Investigation of Prediction Accuracy, Sensitivity, and parameter Stability of Large-Scale Propagation Path loss Models for 5G Wireless Communications," IEEE Transactions on Vehicular Technology, vol. 65, pp. 1-18, May 2016.

[19] S. Li, Y. Liu, L. Lin, X. Sun, S. Yang and D. Sun, "Millimeter-Wave Channel Simulation and Statistical Channel Model in the Cross-Corridor Environment at $28 \mathrm{GHz}$ for 5G Wireless System," 2018 International Conference on Microwave and Millimeter Wave Technology (ICMMT), Chengdu, 2018, pp. 1-3, DoI: 10.1109/ICMMT.2018.8563957.

[20] Q. Faizan, M. H. S. Siddiqui2, M. N. Hindia, and K. Dimyati, "Propagation Channel Measurement at $38 \mathrm{GHz}$ for $5 \mathrm{G} \mathrm{mm}$-wave communication Network," IEEE 16th Student Conference on Research and Development (SCOReD), 2018.

[21] J. S. Seybold, "Introduction to RF propagation,” Wiley, 2005.

[22] T. S. Rappaport, "Wireless communications: principles and practice," Upper Saddle River, N.J.: Prentice Hall PTR, 2 ed., 2002.

[23] Nossire, Z.; Gupta, N.; Almazaydeh, L.; Xiong, X. New Empirical Path Loss Model for $28 \mathrm{GHz}$ and $38 \mathrm{GHz}$ Millimeter Wave in Indoor Urban under Various Conditions. Appl. Sci. 2018, 8, 2122.

[24] ITU-R Recommendation P.1238-10, "Propagation data and prediction methods for the planning of indoor radiocommunication systems and radio local area networks in the frequency range $300 \mathrm{MHz}$ to $450 \mathrm{GHz}$,' 2019.

[25] T. S. Rappaport, E. Ben-Dor, J. N. Murdock, and Y. Qiao “38 GHz and $60 \mathrm{GHz}$ Angle-dependent Propagation for cellular \& Peer-to-Peer Wireless Communications," IEEE ICC 2012 - Wireless Communications Symposium, 2012

[26] M. T. Hagan, H. B. Demuth, M. H. Beale, and O. D. Jesús, "Neural Network Design,” M. Hagan, 2 ed. 2002.

[27] K. P. Murphy, "Machine Learning: A Probabilistic Perspective,” The MIT Press, 2012.

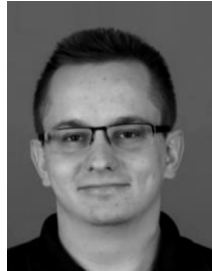

Árpád László Makara is currently an MSc student at the Department of Broadband Infocommunications and Electromagnetic Theory of the Budapest University of Technology and Economics (BME). The topic of his master thesis work is the simulation and optimization of electrostatic ion thrusters for CubeSats. For two semesters, he held exercises at BME for BSc electrical engineering students on the subject of Introduction to Electromagnetic Fields. His current research topic is relating to millimeter wavelength indoor propagation for $5 \mathrm{G}$, and his first results in this field were presented on the EFOP-3.6.2 workshop in November, 2020.

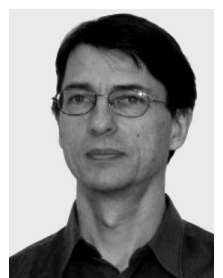

László Csurgai-Horváth is employed as an Associate Professor at Budapest University of Technology and Economics (BME), Department of Broadband Infocommunications and Electromagnetic Theory. His current research interests are focused on indoor and outdoor propagation, measurements and modelling of the rain attenuation on terrestrial and satellite radio links, time series synthesis and cognitive spectrum management. He has been involved in several international projects such as SatNEx, COST Action IC-0802 and QoSMOS. Recently, he has led some ESA-founded research projects relating to the Alphasat propagation and communications experiment and an ESA technology transfer demonstrator project for $5 \mathrm{G}$ indoor propagation measurements. Currently, he teaches space technology and channel modelling at BME in Budapest, Hungary. 